\title{
A STUDY OF SOME RELIABLE METHODS FOR SOLVING FUZZY VOLTERRA-FREDHOLM INTEGRAL EQUATIONS
}

\author{
A.A. Hamoud, K.P. Ghadle
}

ABstract. This paper mainly focuses on the recent advances in the approximated methods for solving fuzzy Volterra-Fredholm integral equations, namely, Adomian decomposition method, modified Adomian decomposition method, variational iteration method and homotopy analysis method. We converted fuzzy VolterraFredholm integral equation of the first kind to the second kind. That, a fuzzy Volterra-Fredholm integral equation has been converted to a system of VolterraFredholm integral equation in crisp case. The approximated methods using to find the approximate solution of this system and hence obtain an approximation for the fuzzy solution of the fuzzy Volterra-Fredholm integral equation. Moreover, we will prove the uniqueness of the solution and convergence of the proposed methods. The study outlines presumed features of these methods as well as sheds some light on advantages of one method over the other. To assess the accuracy of each method, algorithms with Mathematica 6 according is used. Also, some numerical examples are included to demonstrate the validity and applicability of the proposed techniques.

2010 Mathematics Subject Classification: 35A15, 45G15, 65H20, 49M27.

Keywords: Adomian decomposition method, modified Adomian decomposition method, variational iteration method, homotopy analysis method, fuzzy VolterraFredholm integral equation, approximate solution.

\section{INTRODUCTION}

In recent years, the topics of fuzzy integral equations which attracted increasing interest, in particular in relation to fuzzy control, have been rapidly developed. The concept of fuzzy numbers and arithmetic operations firstly introduced by Zadeh $[13,15]$, and then by Dubois and Prade [15]. Also, they have introduced the concept of integration of fuzzy functions. The fuzzy mapping function was introduced by Cheng and Zadeh [13]. Moreover, [14] presented an elementary fuzzy calculus based on the extension principle. Later, Goetschel and Voxman [18] preferred a Riemann 
A.A. Hamoud, K.P. Ghadle - Fuzzy Volterra-Fredholm integral equations ...

integral type approach. Kaleva [19] chose to define the integral of the fuzzy function, using the Lebesgue-type concept for integration. One of the first applications of the fuzzy integral equation was given by $\mathrm{Ma}$ and $\mathrm{Wu}$ who investigated the fuzzy Fredholm integral equation of the second kind. Recently, some mathematicians have studied fuzzy integral and integro-differential equation by numerical techniques $[6,8,9,12,17,29]$. As we know the fuzzy integral and differential equations are one of the important parts of the fuzzy analysis theory that play a main role in the numerical analysis.

In this work, we will suggest recent advances in the approximated methods for solving fuzzy Volterra-Fredholm integral equations of the first and second kind, namely, Adomian decomposition method, modified decomposition method, variational iteration method and homotopy analysis method.

The rest of the paper is organized as follows: In Section 2, we will discuss the basic concepts of basic notations and definitions in fuzzy calculus. In Section 3, the fuzzy Volterra-Fredholm integral equation of the first kind is briefly presented and we will convert fuzzy Volterra-Fredholm integral equation of the first kind to the second kind. In Section 4, we will convert a fuzzy Volterra-Fredholm integral equation of the second kind to the system of Volterra-Fredholm integral equation of the second kind in a crisp case. In Section 5, we will highlight briefly on some reliable methods for solving this type of equations. In Section 6, we will prove the uniqueness of the solution and convergence of the methods. In Section 7, the numerical examples are presented to illustrate the accuracy of these methods. In Section 8, we will discuss the results and comparison among these methods. Finally, we will give a report on our paper and a brief conclusion are given in Section 9 .

\section{BASIC CONCEPTS}

The concept of fuzzy numbers is generalized of classical real numbers and we can say that a fuzzy number is a fuzzy subset of the real line which has some additional properties. The concept of fuzzy number is vital for fuzzy analysis, fuzzy integral equations and fuzzy differential equations, and a very helpful tool in different applications of fuzzy sets. Basic definition of fuzzy numbers is given in $[3,11,13,15]$.

Definition 1. A fuzzy number is a fuzzy set like $u: \mathbb{R} \rightarrow[0,1]$ with the following properties:

- $u$ is upper semi-continuous function,

- $u$ is fuzzy convex,i.e, $u(\lambda x+(1-\lambda) y) \geq \min \{u(x), u(y)\}$ for all $x, y \in \mathbb{R}, \lambda \in$ $[0,1]$, 
- $u$ is normal, i.e, $\exists x_{0} \in \mathbb{R}$ for which $u\left(x_{0}\right)=1$,

- $\sup u=\{x \in \mathbb{R} \mid u(x)>0\}$ is the support of the $u$, and its closure $c l(\sup u)$ is compact.

Let $\mathbb{E}$ be the set of all fuzzy numbers on $\mathbb{R}$. The $(\alpha-c u t) \alpha$-level set of a fuzzy number $u \in \mathbb{E}, 0 \leq \alpha \leq 1$, denoted by $[u]_{\alpha}$, is defined as

$$
[u]_{\alpha}= \begin{cases}\{x \in \mathbb{R}: u(x) \geq \alpha\}, & 0<\alpha \leq 1 \\ c l(\sup u), & \alpha=0\end{cases}
$$

where $c l(\sup u=x \in \mathbb{R} \mid u(x)>0)$ denotes the closure of the support of $u$. It is clear that the $\alpha$-level set of a fuzzy number is a closed and bounded interval $[\underline{u}(\alpha), \bar{u}(\alpha)]$, where $\underline{u}(\alpha)$ denotes the left-hand end point of $[u]_{\alpha}$ and $\bar{u}(\alpha)$ denotes the right-hand end point of $[u]_{\alpha}$. Since each $u \in \mathbb{R}$ can be regarded as a fuzzy number $\tilde{u}$ defined by:

$$
\tilde{u}(t)= \begin{cases}1, & t=u \\ 0, & t \neq u\end{cases}
$$

An equivalent parametric definition is also given in [13] as:

Definition 2. A fuzzy number $\tilde{u}$ in parametric form is a pair $(\underline{u}, \bar{u})$ of functions $\underline{u}(\alpha), \bar{u}(\alpha), 0 \leq \alpha \leq 1$, which satisfy the following requirements:

- $\underline{u}(\alpha)$ is a bounded non-decreasing left continuous function in $(0,1]$, and right continuous at 0 ,

- $\bar{u}(\alpha)$ is a bounded non-increasing left continuous function in $(0,1]$, and right continuous at 0 ,

- $\underline{u}(\alpha) \leq \bar{u}(\alpha), \quad 0 \leq \alpha \leq 1$.

A crisp number $\alpha$ is simply represented by $\underline{u}(\alpha)=\bar{u}(\alpha)=\alpha, 0 \leq \alpha \leq 1$.

We recall that for $a<b<c$ which $a, b, c \in \mathbb{R}$, the triangular fuzzy number $u=(a, b, c)$ determined by $a, b, c$ are given such that $\underline{u}(\alpha)=a+(b-a) \alpha$ and $\bar{u}(\alpha)=c-(c-b) \alpha$ are the end points of the $\alpha$-level sets, for all $\alpha \in[0,1]$.

The Hausdorff distance between fuzzy numbers given by $d: \mathbb{E} \times \mathbb{E} \rightarrow \mathbb{R}_{+} \cup\{0\}$.

$$
d(u, v)=\sup _{\alpha \in[0,1]} \max \{|\underline{u}(\alpha)-\underline{v}(\alpha)|,|\bar{u}(\alpha)-\bar{v}(\alpha)|\},
$$

wehre $u=(\underline{u}(\alpha), \bar{u}(\alpha)), v=(\underline{v}(\alpha), \bar{v}(\alpha)) \subset \mathbb{R}$ is utilized in [13]. Then, it is easy to see that $d$ is a metric in $\mathbb{E}$ and has the following properties [11]: 
- $d(u+\rho, v+\rho)=d(u, v), \quad \forall u, v, \rho \in \mathbb{E}$,

- $d(k u, k v)=|k| d(u, v), \quad \forall k \in \mathbb{R} ; u, v \in E$,

- $d(\omega+v, \rho+e) \leq d(\omega, \rho)+d(v, e), \quad \forall \omega, v, \rho, e \in \mathbb{E}$,

- $(d, \mathbb{E})$ is a complete metric space.

Definition 3. [11] Let $f: \mathbb{R} \rightarrow \mathbb{E}$ be a fuzzy valued function. If for arbitrary fixed $t_{0} \in R$ and $\forall \epsilon>0, \exists \delta>0$ such that $\left|t-t_{0}\right|<\delta \Longrightarrow\left|f(t)-f\left(t_{0}\right)\right|<\epsilon$, then $f$ is said to be continuous.

Theorem 1. [11] Let $f(x)$ be a fuzzy-valued function on $[a, \infty)$ and it is represented by $(f(x, \alpha), \bar{f}(x, \alpha))$. For any fixed $t \in[0,1]$ assume $f(x, \alpha)$ and $\bar{f}(x, \alpha)$ are Riemann-integrable on $[a, b]$ for every $b \geq a$, and assume there are two positive $\underline{M}(\alpha)$ and $\bar{M}(\alpha)$ such that $\int_{a}^{b}|\underline{f}(x, \alpha)| d x \leq \underline{M}(\alpha)$ and $\int_{a}^{b}|\bar{f}(x, \alpha)| d x \leq \bar{M}(\alpha)$ for every $b \geq a$. Then $f(x)$ is improper fuzzy Riemann-integrable on $[a, \infty)$ and the improper fuzzy Riemann-integral is a fuzzy number. Furthermore, we have:

$$
\int_{a}^{\infty} f(x) d x=\left(\int_{a}^{\infty} \underline{f}(x, \alpha) d x, \int_{a}^{\infty} \bar{f}(x, \alpha) d x\right) .
$$

Proposition 1. [27]. If each of $f(x)$ and $g(x)$ is fuzzy-valued function and fuzzy Riemman integrable on $\Omega=[a, \infty)$ then $f(x)+g(x)$ is fuzzy Riemman integrable on $\Omega$. Moreover, we have:

$$
\int_{\Omega}(f(x)+g(x)) d x=\int_{\Omega} f(x) d x+\int_{\Omega} g(x) d x .
$$

Definition 4. [9] The integral of a fuzzy function was define by using the Riemann integral concept. Let $f:[a, b] \rightarrow \mathbb{E}$, for each partition $P=t_{0}, t_{1}, \ldots, t_{n}$ of $[a, b]$ and for arbitrary $\xi_{i} \in\left[t_{i-1}, t_{i}\right], 1 \leq i \leq n$, suppose

$$
\begin{gathered}
R_{p}=\sum_{i=1}^{n} f\left(\xi_{i}\right)\left(t_{i}-t_{i-1}\right) . \\
\Delta:=\max \left|t_{i}-t_{i-1}\right|, 1 \leq i \leq n .
\end{gathered}
$$

The definite integral of $f(t)$ over $[a, b]$ is

$$
\int_{a}^{b} f(t) d t=\lim _{\Delta \rightarrow 0} R_{p}
$$

Provided that this limit exists in the metric d. If the fuzzy function $f(t)$ is continuous in the metric d, its definite integral exists [17], and also 


$$
\begin{aligned}
& \underline{\int_{a}^{b} f(t, r)} d t=\int_{a}^{b} \underline{f}(t, r) d t \\
& \overline{\int_{a}^{b} f(t, r)} d t=\int_{a}^{b} \bar{f}(t, r) d t
\end{aligned}
$$

It should be noted that the fuzzy integral can be also defined using the Lebesguetype approach [3]. However, if $f(t)$ is continuous, both approaches yield the same value. More details about the properties of the fuzzy integral are given in $[15,29,27]$.

\section{Fuzzy Volterra-Fredholm Integral Equations of the First Kind}

We consider the fuzzy Volterra-Fredholm integral equation of the first kind given by

$$
\tilde{f}(x)=\lambda_{1} \int_{a}^{x} k_{1}(x, t) G_{1}(\tilde{u}(t)) d t+\lambda_{2} \int_{a}^{b} k_{2}(x, t) G_{2}(\tilde{u}(t)) d t,
$$

where $a, b, \lambda_{1}$ and $\lambda_{2}$ are constant values and $\lambda_{1} \neq 0$, also $f(x), k_{1}(x, t), k_{2}(x, t), G_{1}(\tilde{u}(t))$ and $G_{2}(\tilde{u}(t))$ are functions that have suitable derivatives on an interval $a \leq t \leq x \leq b$ and $\tilde{u}(t)$ is unknown function. The solution is expressed in the form [9]:

$$
\tilde{u}(x)=\sum_{i=0}^{\infty} \tilde{u}_{i}(x) .
$$

We reduce integral equation of the first kind to the second kind by differentiating one time with respect to $x$. Consider the nonlinear Volterra-Fredholm integral equation of the first kind Eq.(1) where $\tilde{u}(t)$ is an unknown function, and $\tilde{f}(x), k_{1}(x, t)$ and $k_{2}(x, t)$ are analytical functions. To obtain the solution of equation Eq.(1) in the form of expression Eq.(2) we first differentiate it one time with respect to $x$ :

$\tilde{f}^{\prime}(x)=\lambda_{1} k_{1}(x, x) G_{1}(\tilde{u}(x))+\lambda_{1} \int_{a}^{x} \frac{\partial k_{1}(x, t)}{\partial x} G_{1}(\tilde{u}(t)) d t+\lambda_{2} \int_{a}^{b} \frac{\partial k_{2}(x, t)}{\partial x} G_{2}(\tilde{u}(t)) d t$,

Since $k_{1}(x, x) \neq 0$, therefore,

$$
G_{1}(\tilde{u}(x))=\frac{\tilde{f}^{\prime}(x)}{\lambda_{1} k_{1}(x, x)}-\int_{a}^{x} \frac{\frac{\partial k_{1}(x, t)}{\partial x}}{k_{1}(x, x)} G_{1}(\tilde{u}(t)) d t-\frac{\lambda_{2}}{\lambda_{1}} \int_{a}^{b} \frac{\frac{\partial k_{2}(x, t)}{\partial x}}{k_{1}(x, x)} G_{2}(\tilde{u}(t)) d t,
$$


we set

$$
\begin{aligned}
& \tilde{F}(x)=\frac{\tilde{f}^{\prime}(x)}{\lambda_{1} k_{1}(x, x)}, \\
& K_{1}^{\prime}(x, t)=\left(\frac{\frac{\partial k_{1}(x, t)}{\partial x}}{k_{1}(x, x)}\right), \\
& K_{2}^{\prime}(x, t)=-\frac{\lambda_{2}}{\lambda_{1}} \frac{\frac{\partial k_{2}(x, t)}{\partial x}}{k_{1}(x, x)} .
\end{aligned}
$$

Therefore,

$$
\tilde{u}(x)=G_{1}^{-1}(\tilde{F}(x))+G_{1}^{-1}\left(\int_{a}^{x} K_{1}^{\prime}(x, t) G_{1}(\tilde{u}(t)) d t\right)+G_{1}^{-1}\left(\int_{a}^{b} K_{2}^{\prime}(x, t) G_{2}(\tilde{u}(t)) d t\right),
$$

where $K_{1}^{\prime}(x, t), K_{2}^{\prime}(x, t)$ and $G_{1}^{-1}(\tilde{F}(x))$ are functions that have suitable derivatives on an interval $a \leq t \leq x \leq b$. So, Eq.(1) reduces to the standard VolterraFredholm integral equation of the second kind.

\section{Fuzzy Volterra-Fredholm integral equation of the Second Kind}

The fuzzy Volterra-Fredholm integral equation of the second kind is as follows:

$$
\tilde{u}(x)=\tilde{f}(x)+\mu_{1} \int_{a}^{x} K_{1}(x, t) G_{1}(t, \tilde{u}(t)) d t+\mu_{2} \int_{a}^{b} K_{2}(x, t) G_{2}(t, \tilde{u}(t)) d t,
$$

where $\mu_{1}, \mu_{2} \geq 0, \tilde{f}(x)$ is a fuzzy function of $x: a \leq x \leq b$, and $K_{i}(x, t), G_{i}(t, \tilde{u}(t)), i=$ 1,2 , are analytic functions on $[a, b]$. For solving in parametric form of Eq.(6), consider $(f(x, r), f(x, r))$ and $(u(x, r), u(x, r)), 0 \leq r \leq 1$ and $t \in[a, b]$ are parametric form of $\tilde{f}(x)$ and $\tilde{u}(x)$, respectively. Then, parametric form of Eq.(6) is as follows:

$$
\begin{aligned}
& \underline{u}(x, r)=\underline{f}(x, r)+\mu_{1} \int_{a}^{x} \underline{K_{1}(x, t) G_{1}(t, u(t, r))} d t+\mu_{2} \int_{a}^{b} \underline{K_{2}(x, t) G_{2}(t, u(t, r))} d t, \\
& \bar{u}(x, r)=\bar{f}(x, r)+\mu_{1} \int_{a}^{x} \overline{K_{1}(x, t) G_{1}(t, u(t, r))} d t+\mu_{2} \int_{a}^{b} \overline{K_{2}(x, t) G_{2}(t, u(t, r))} d t .
\end{aligned}
$$

Let for $a \leq t \leq b$, we have

$$
\begin{aligned}
& H_{1}(t, \underline{u}, \bar{u})=\min \left\{G_{1}(t, \beta) \mid \underline{u}(t, r) \leq \beta \leq \bar{u}(t, r)\right\}, \\
& H_{2}(t, \underline{u}, \bar{u})=\min \left\{G_{2}(t, \beta) \mid \underline{u}(t, r) \leq \beta \leq \bar{u}(t, r)\right\}, \\
& F_{1}(t, \underline{u}, \bar{u})=\max \left\{G_{1}(t, \beta) \mid \underline{u}(t, r) \leq \beta \leq \bar{u}(t, r)\right\}, \\
& F_{2}(t, \underline{u}, \bar{u})=\max \left\{G_{2}(t, \beta) \mid \underline{u}(t, r) \leq \beta \leq \bar{u}(t, r)\right\} .
\end{aligned}
$$


Then,

$$
\begin{aligned}
& \underline{K_{1}(x, t) G_{1}(t, u(t, r))}= \begin{cases}K_{1}(x, t) H_{1}(t, \underline{u}, \bar{u}), & K_{1}(x, t) \geq 0 \\
K_{1}(x, t) F_{1}(t, \underline{u}, \bar{u}), & K_{1}(x, t)<0 .\end{cases} \\
& \underline{K_{2}(x, t) G_{2}(t, u(t, r))}= \begin{cases}K_{2}((x, t)) H_{2}(t, \underline{u}, \bar{u}), & K_{2}(x, t) \geq 0, \\
K_{2}((x, t)) F_{2}(t, \underline{u}, \bar{u}), & K_{2}(x, t)<0 .\end{cases} \\
& \overline{K_{1}(x, t) G_{1}(t, u(t, r))}= \begin{cases}K_{1}(x, t) F_{1}(t, \underline{u}, \bar{u}), & K_{1}(x, t) \geq 0 \\
K_{1}(x, t) H_{1}(t, \underline{u}, \bar{u}), & K_{1}(x, t)<0 .\end{cases} \\
& \overline{K_{2}(x, t) G_{2}(t, u(t, r))}= \begin{cases}K_{2}(x, t) F_{2}(t, \underline{u}, \bar{u}), & K_{2}(x, t) \geq 0, \\
K_{2}(x, t) H_{2}(t, \underline{u}, \bar{u}), & K_{2}(x, t)<0 .\end{cases}
\end{aligned}
$$

For each $0 \leq r \leq 1$ and $a \leq x \leq b$. We can see that Eq.(6) convert to a system of Volterra-Fredholm integral equations in crisp case for each $0 \leq r \leq 1$ and $a \leq t \leq b$. Now, we explain Adomian decomposition method and modified Adomian decomposition method for approximating solution of this system of integral equations in crisp case. Then, we find approximate solutions for $\tilde{u}(x), a \leq x \leq b$.

\section{Description of the Methods}

Here we will highlight briefly on some reliable methods for solving this type of equations, where details can be found in $[2,3,4,5,6,7,26,28]$.

\subsection{Adomian Decomposition Method (ADM)}

Firstly, we explain Adomian decomposition method. The Adomian decomposition method has been applied to a wild class of functional equations $[2,7,8,10,16,21,22]$ by scientists and engineers since the beginning of the 1980s. Adomian gives the solution as a infinite series usually converging to a solution consider the following fuzzy Volterra-Fredholm integral equation of the form

$$
\begin{aligned}
\underline{u}(x, r) & =\underline{f}(x, r)+\mu_{1} \int_{a}^{x} \frac{K_{1}(x, t) G_{1}(t, u(t, r))}{x} d t+\mu_{2} \int_{a}^{b} \frac{K_{2}(x, t) G_{2}(t, u(t, r))}{b} d t \\
\bar{u}(x, r) & =\bar{f}(x, r)+\mu_{1} \int_{a}^{x} \overline{K_{1}(x, t) G_{1}(t, u(t, r))} d t+\mu_{2} \int_{a}^{b} \overline{K_{2}(x, t) G_{2}(t, u(t, r))} d t
\end{aligned}
$$


The ADM assume an infinite series solution for the unknowns functions $[\underline{u}, \bar{u}]$, given by

$$
\begin{aligned}
& \underline{u}(x)=\sum_{i=0}^{\infty} \underline{u}_{i}(x), \\
& \bar{u}(x)=\sum_{i=0}^{\infty} \bar{u}_{i}(x) .
\end{aligned}
$$

The nonlinear operators $G_{1}(t, \underline{u}(t)), G_{1}(t, \bar{u}(t)), G_{2}(t, \underline{u}(t))$ and $G_{2}(t, \bar{u}(t))$ into an infinite series of polynomials given by

$$
\begin{array}{ll}
G_{1}(t, \underline{u}(t))=\sum_{i=0}^{\infty} \underline{A}_{n}, & G_{1}(t, \bar{u}(t))=\sum_{i=0}^{\infty} \bar{A}_{n}, \\
G_{2}(t, \underline{u}(t))=\sum_{i=0}^{\infty} \underline{B}_{n}, & G_{2}(t, \bar{u}(t))=\sum_{i=0}^{\infty} \bar{B}_{n} .
\end{array}
$$

where the $\tilde{A}_{n}=\left[\underline{A}_{n}, \bar{A}_{n}\right], \tilde{B}_{n}=\left[\underline{B}_{n}, \bar{B}_{n}\right], n \geq 0$, are the so-called Adomian polynomial. Substituting Eqs.(10) and Eqs.(11) into Eq.(9), we get

$$
\begin{aligned}
& \underline{u}_{0}=\underline{f}(x, r), \\
& \underline{u}_{1}=\mu_{1} \int_{a}^{x} \underline{K_{1}(x, t) A_{0}} d t+\mu_{2} \int_{a}^{b} \underline{K_{2}(x, t) B_{0}} d t, \\
& \text {. } \\
& \text {. } \\
& \underline{u}_{n+1}=\mu_{1} \int_{a}^{x} \underline{K_{1}(x, t) A_{n}} d t+\mu_{2} \int_{a}^{b} \underline{K_{2}(x, t) B_{n}} d t .
\end{aligned}
$$

and

$$
\begin{aligned}
& \bar{u}_{0}=\bar{f}(x, r), \\
& \bar{u}_{1}=\mu_{1} \int_{a}^{x} \overline{K_{1}(x, t) A_{0}} d t+\mu_{2} \int_{a}^{b} \overline{K_{2}(x, t) B_{0}} d t, \\
& \cdot \\
& \bar{u}_{n+1}=\mu_{1} \int_{a}^{x} \overline{K_{1}(x, t) A_{n}} d t+\mu_{2} \int_{a}^{b} \overline{K_{2}(x, t) B_{n}} d t .
\end{aligned}
$$

We approximate $\tilde{u}(x, r)=[\underline{u}(x, r), \bar{u}(x, r)]$ by 


$$
\begin{aligned}
\underline{\varphi}_{n} & =\sum_{i=0}^{n-1} \underline{u}_{i}(x, r), \\
\bar{\varphi}_{n} & =\sum_{i=0}^{n-1} \bar{u}_{i}(x, r),
\end{aligned}
$$

where,

$$
\lim _{n \rightarrow \infty} \underline{\varphi}_{n}=\underline{u}(x, r), \lim _{n \rightarrow \infty} \bar{\varphi}_{n}=\bar{u}(x, r) .
$$

Now, we explain modified Adomian decomposition method.

\subsection{Modified Adomian Decomposition Method (MADM)}

The modified Adomian decomposition method was introduced by Wazwaz [30]. This method is based on the assumption that the function $f(x)$ can be divided into two parts, namely $f_{1}(x)$ and $f_{2}(x)$. Under this assumption we set

$$
f(x)=f_{1}(x)+f_{2}(x) .
$$

We apply this decomposition when the function $f(x)$ consists of several parts and can be decomposed into two different parts $[6,4]$. In this case, $f(x)$ is usually a summation of a polynomial and trigonometric or transcendental functions. A proper choice for the part $f_{1}$ is important. For the method to be more efficient, we select $f_{1}$ as one term of $f(x)$ or at least a number of terms if possible and $f_{2}$ consists of the remaining terms of $f(x)$. In comparison with the standard decomposition method, the MADM minimizes the size of calculations and the cost of com putational operations in the algorithm. Both standard and modified decomposition methods are reliable for solving nonlinear problems such as Volterra-Fredholm integro differential equations, but in order to decrease the complexity of the algorithm and simplify the calculations we prefer to use the MDM. The MDM will accelerate the rapid convergence of the series solution in comparison with the standard Adomian decomposition method. The modified technique may give the exact solution for nonlinear equations without the necessity to find the Adomian polynomials. We refer the reader to $[23,24,30]$ for more details about the MADM. Accordingly, a slight variation was proposed only on the components $u_{0}$ and $u_{1}$. The suggestion was that only the part $f_{1}$ be assigned to the component $u_{0}$, whereas the remaining part $f_{2}$ be combined with the other terms to define $u_{1}$. Consequently, the following modified recursive relation was developed: 
we get

$$
\begin{aligned}
& \underline{u}_{0}=\underline{f}_{1}(x, r), \\
& \underline{u}_{1}=\underline{f}_{2}(x, r)+\mu_{1} \int_{a}^{x} \underline{K_{1}(x, t) A_{0}} d t+\mu_{2} \int_{a}^{b} \underline{K_{2}(x, t) B_{0}} d t, \\
& \cdot \\
& \text {. } \\
& \underline{u}_{n+1}=\mu_{1} \int_{a}^{x} \underline{K_{1}(x, t) A_{n}} d t+\mu_{2} \int_{a}^{b} \underline{K_{2}(x, t) B_{n}} d t, n \geq 1 .
\end{aligned}
$$

and

$$
\begin{aligned}
& \bar{u}_{0}=\bar{f}_{1}(x, r), \\
& \bar{u}_{1}=\bar{f}_{2}(x, r)+\mu_{1} \int_{a}^{x} \overline{K_{1}(x, t) A_{0}} d t+\mu_{2} \int_{a}^{b} \overline{K_{2}(x, t) B_{0}} d t \\
& \cdot \\
& \cdot \\
& \bar{u}_{n+1}=\mu_{1} \int_{a}^{x} \overline{K_{1}(x, t) A_{n}} d t+\mu_{2} \int_{a}^{b} \overline{K_{2}(x, t) B_{n}} d t, n \geq 1 .
\end{aligned}
$$

We approximate $\tilde{u}(x, r)=[\underline{u}(x, r), \bar{u}(x, r)]$ by

$$
\begin{aligned}
& \underline{\varphi}_{n}=\sum_{i=0}^{n-1} \underline{u}_{i}(x, r), \\
& \bar{\varphi}_{n}=\sum_{i=0}^{n-1} \bar{u}_{i}(x, r),
\end{aligned}
$$

where,

$$
\lim _{n \rightarrow \infty} \underline{\varphi}_{n}=\underline{u}(x, r), \lim _{n \rightarrow \infty} \bar{\varphi}_{n}=\bar{u}(x, r)
$$

\subsection{Variational Iteration Method (VIM)}

The variational iteration method (VIM) is proposed by (He 1997) [20, 28] as a modification of a general Lagrange multiplier method. This method has been shown to solve effectively, easily and accurately a large class of nonlinear problems with 
approximations converging rapidly to a accurate solutions. To illustrate its basic idea of the technique, we consider following general nonlinear system:

$$
L[u(x)]+N[u(x)]=g(x),
$$

Where $L$ is linear operator, $N$ is a nonlinear operator, and $g(x)$ is given continuous function. The basic character of method is to a correction functional for system Eq.(17) which

$$
u_{n+1}(x)=u_{n}(x)+\int_{0}^{x} \lambda(\tau)\left\{L u_{n}(\tau)+N \tilde{u}_{n}(\tau)-g(\tau)\right\} d \tau,
$$

Where $\lambda(\tau)$ is a general Lagrangian multiplier (Kaleva 1987) which can be identified optimally via variational theory, the subscript $n$ denotes the $n^{\text {th }}$-order approximation and $\tilde{u}_{n}$ is consider a restricted variation, i.e. $\delta \tilde{u}_{n}=0$ where $L=\frac{d}{d t}$. For the integral equation (6), let $w(x)$ be a function such that $w^{\prime}(x)=\tilde{u}(x)$, noting that $\tilde{u}(x)$ is continuous. Then we have

$$
w^{\prime}(x)=\tilde{f}(x)+\mu_{1} \int_{a}^{x} K_{1}(x, t) G_{1}\left(t, w^{\prime}(t)\right) d t+\mu_{2} \int_{a}^{b} K_{2}(x, t) G_{2}\left(t, w^{\prime}(t)\right) d t .
$$

Consider

$$
\mu_{1} \int_{a}^{x} K_{1}(x, t) G_{1}\left(t, w^{\prime}(t)\right) d t+\mu_{2} \int_{a}^{b} K_{2}(x, t) G_{2}\left(t, w^{\prime}(t)\right) d t
$$

as a restricted variation; we have the iteration sequence

$$
w_{n+1}=w_{n}+\int_{0}^{x} \lambda\left[w_{n}^{\prime}(s)-\mu_{1} \int_{a}^{s} K_{1}(s, t) G_{1}\left(t, w^{\prime}(t)\right) d t-\mu_{2} \int_{a}^{b} K_{2}(s, t) G_{2}\left(t, w^{\prime}(t)\right) d t-\tilde{f}(s)\right] d s .
$$

Taking the variation with respect to the independent variable $w_{n}$ and noticing that $\delta w_{n}(0)=0$, we get

$$
\delta w_{n+1}=\delta w_{n}+\left.\lambda(s) \delta w_{n}\right|_{s=x}-\int_{0}^{x} \lambda^{\prime}(s) \delta w_{n} d s=0
$$

Then we apply the following stationary conditions:

$$
1+\left.\lambda(s)\right|_{s=x}=0,\left.\quad \lambda^{\prime}(s)\right|_{s=x}=0,
$$

The general Lagrange multiplier, therefore, can be readily identified:

$$
\lambda=-1
$$

and, as a result, we obtain the following iteration formula: 
$w_{n+1}=w_{n}-\int_{0}^{x}\left[w_{n}^{\prime}(s)-\tilde{f}(s)-\mu_{1} \int_{a}^{s} K_{1}(s, t) G_{1}\left(t, w^{\prime}(t)\right) d t-\mu_{2} \int_{a}^{b} K_{2}(s, t) G_{2}\left(t, w^{\prime}(t)\right) d t\right] d s$.

Therefore, we can write the following iteration formulas

$$
\begin{aligned}
\underline{u}_{n+1}(x, r)= & \underline{u}_{n}(x, r)-\int_{0}^{x} \\
& {\left[\underline{u}_{n}(s, r)-\underline{f}(s, r)-\mu_{1} \int_{a}^{s} K_{1}(s, t) G_{1}(t, \underline{u}(t, r)) d t-\mu_{2} \int_{a}^{b} K_{2}(s, t) G_{2}(t, \underline{u}(t, r)) d t\right] d s . } \\
\bar{u}_{n+1}(x, r)= & \bar{u}_{n}(x, r)-\int_{0}^{x} \\
& {\left[\bar{u}_{n}(s, r)-\bar{f}(s, r)-\mu_{1} \int_{a}^{s} K_{1}(s, t) G_{1}(t, \bar{u}(t, r)) d t-\mu_{2} \int_{a}^{b} K_{2}(s, t) G_{2}(t, \bar{u}(t, r)) d t\right] d s . }
\end{aligned}
$$

\subsection{Homotopy Analysis Method (HAM)}

Consider,

$$
N[\tilde{u}]=0,
$$

where $N$ is a nonlinear operator, $\tilde{u}=[\underline{u}(x, r), \bar{u}(x, r)]$ are unknown functions and $x$ is an independent variable. Let $\underline{u}_{0}(x, r), \bar{u}_{0}(x, r)$ denote an initial guess of the exact solution $\underline{u}(x, r), \bar{u}(x, r), h \neq 0$ an auxiliary parameter, $H_{1}(x) \neq 0$ an auxiliary function, and $L$ an auxiliary linear operator with the property $L[s(x)]=0$ when $s(x)=0$. Then using $q \in[0,1]$ as an embedding parameter [25], we can construct a homotopy when consider, $N[\underline{u}]=0$, as follows:

$(1-q) L\left[\underline{\phi}(x ; q, r)-\underline{u}_{0}(x, r)\right]-q h H_{1}(x) N[\phi(x ; q, r)]=\hat{H}\left[\phi(x ; q, r) ; \underline{u}_{0}(x, r), H_{1}(x), h, q\right]$.

It should be emphasized that we have great freedom to choose the initial guess $\underline{u}_{0}(x, r)$, the auxiliary linear operator $L$, the non-zero auxiliary parameter $h$, and the auxiliary function $H_{1}(x)$. Enforcing the homotopy Eq.(24) to be zero, i.e.,

$$
\hat{H}_{1}\left[\underline{\phi}(x ; q, r) ; \underline{u}_{0}(x, r), H_{1}(x), h, q\right]=0,
$$

we have the so-called zero-order deformation equation

$$
(1-q) L\left[\underline{\phi}(x ; q, r)-\underline{u}_{0}(x, r)\right]=q h H_{1}(x) N[\phi(x ; q, r)],
$$

when $q=0$, the zero-order deformation Eq.(26) becomes

$$
\underline{\phi}(x ; 0, r)=\underline{u}_{0}(x, r),
$$


and when $q=1$, since $h \neq 0$ and $H_{1}(x) \neq 0$, the zero-order deformation Eq.(26) is equivalent to

$$
\underline{\phi}(x ; 1, r)=\underline{u}(x, r) .
$$

Thus, according to Eqs.(27) and (28), as the embedding parameter $q$ increases from 0 to $1, \phi(x ; q, r)$ varies continuously from the initial approximation $\underline{u}_{0}(x, r)$ to the exact solution $\underline{u}(x, r)$. Such a kind of continuous variation is called deformation in homotopy. Due to Taylor's theorem, $\phi(x ; q, r)$ can be expanded in a power series of $q$ as follows

$$
\underline{\phi}(x ; q, r)=\underline{u}_{0}(x, r)+\sum_{m=1}^{\infty} \underline{u}_{m}(x, r) q^{m}
$$

where,

$$
\underline{u}_{m}(x, r)=\left.\frac{1}{m !} \frac{\partial^{m} \underline{\phi}(x ; q, r)}{\partial q^{m}}\right|_{q=0} .
$$

Let the initial guess $\underline{u}_{0}(x, r)$, the auxiliary linear parameter $L$, the nonzero auxiliary parameter $h$ and the auxiliary function $H_{1}(x)$ be properly chosen so that the power series Eq. $(29)$ of $\phi(x ; q, r)$ converges at $q=1$, then, we have under these assumptions the solution series

$$
\underline{u}(x, r)=\underline{\phi}(x ; 1, r)=\underline{u}_{0}(x, r)+\sum_{m=1}^{\infty} \underline{u}_{m}(x, r) .
$$

From Eq.(29), we can write Eq.(26) as follows:

$$
\begin{aligned}
(1-q) L\left[\underline{\phi}(x ; q, r)-\underline{u}_{0}(x, r)\right] & =(1-q) L\left[\sum_{m=1}^{\infty} \underline{u}_{m}(x, r) q^{m}\right] \\
& =q h H_{1}(x) N[\underline{\phi}(x ; q, r)],
\end{aligned}
$$

then,

$$
L\left[\sum_{m=1}^{\infty} \underline{u}_{m}(x, r) q^{m}\right]-q L\left[\sum_{m=1}^{\infty} \underline{u}_{m}(x, r) q^{m}\right]=q h H_{1}(x) N[\underline{\phi}(x ; q, r)] .
$$

By differentiating Eq.(32) $\mathrm{m}$ times with respect to $q$, we obtain

$$
\begin{aligned}
\left\{L\left[\sum_{m=1}^{\infty} \underline{u}_{m}(x, r) q^{m}\right]-q L\left[\sum_{m=1}^{\infty} \underline{u}_{m}(x, r) q^{m}\right]\right\}^{(m)} & =q h H_{1}(x) N[\underline{\phi}(x ; q, r)]^{(m)} \\
& =m ! L\left[\underline{u}_{m}(x)-\underline{u}_{m-1}(x, r)\right] \\
& =\left.h H_{1}(x) m \frac{\partial^{m-1} N[\underline{\phi}(x ; q, r)]}{\partial q^{m-1}}\right|_{q=0} .
\end{aligned}
$$


A.A. Hamoud, K.P. Ghadle - Fuzzy Volterra-Fredholm integral equations ...

Therefore,

$$
L\left[\underline{u}_{m}(x, r)-\chi_{m} \underline{u}_{m-1}(x, r)\right]=h H_{1}(x) \Re_{m}\left(\underline{u}_{m-1}(x, r)\right),
$$

where,

$$
\Re_{m}\left(\underline{u}_{m-1}(x)\right)=\left.\frac{1}{(m-1) !} \frac{\partial^{m-1} N[\varphi(x ; q)]}{\partial q^{m-1}}\right|_{q=0}
$$

and

$$
\chi_{m}= \begin{cases}0 & m \leq 1, \\ 1 & m>1 .\end{cases}
$$

Note that the high-order deformation Eq.(34) is governing the linear operator $L$, and the term $\Re_{m}\left(\underline{u}_{m-1}(x, r)\right)$ can be expressed simply by Eq.(35) for any nonlinear operator $N$.

To obtain the approximation solution of Eq.(7), according to HAM, let us do $N[\underline{u}(x, r)]=\underline{u}(x, r)-\underline{f}(x, r)-\mu_{1} \int_{a}^{x} \underline{K_{1}(x, t) G_{1}(t, u(t, r))} d t-\mu_{2} \int_{a}^{b} \underline{K_{2}(x, t) G_{2}(t, u(t, r))} d t$.

So,

$$
\begin{aligned}
& \Re_{m}\left(\underline{u}_{m-1}(x, r)\right)=\underline{u}_{m-1}(x, r)-\underline{f}(x, r)-\mu_{1} \int_{a}^{x} \underline{K_{1}(x, t) G_{1}(t, u(t, r))} d t \\
&-\mu_{2} \int_{a}^{b} \underline{K_{2}(x, t) G_{2}(t, u(t, r))} d t-\left(1-\chi_{m}\right) \underline{f}(x, r), m \geq 1 .
\end{aligned}
$$

Substituting Eq.(36) into Eq. (34)

$$
\begin{aligned}
& L\left[\underline{u}_{m}(x, r)-\chi_{m} \underline{u}_{m-1}(x, r)\right]=h H_{1}(x)\left[\underline{u}_{m-1}(x, r)-\mu_{1} \int_{a}^{x} \underline{K_{1}(x, t) G_{1}(t, u(t, r))} d t\right. \\
& \left.-\mu_{2} \int_{a}^{b} \underline{K_{2}(x, t) G_{2}(t, u(t, r))} d t-\left(1-\chi_{m}\right) \underline{f}(x, r)\right] .
\end{aligned}
$$

We take an initial guess $\underline{u}_{0}(x, r)=f(x, r)$, an auxiliary linear operator $L \underline{u}=\underline{u}$, a nonzero auxiliary parameter $h=-1$, and auxiliary function $H_{1}(x)=1$. This is substituted into Eq.(37) to give the recurrence relation

$$
\begin{aligned}
\underline{u}_{0}(x, r) & =\underline{f}(x, r) \\
\underline{u}_{n+1}(x, r) & =\mu_{1} \int_{a}^{x} \underline{K_{1}(x, t) G_{1}\left(t, u_{n}(t, r)\right)} d t+\mu_{2} \int_{a}^{b} \underline{K_{2}(x, t) G_{2}\left(t, u_{n}(t, r)\right)} d t, n \geq 0
\end{aligned}
$$


A.A. Hamoud, K.P. Ghadle - Fuzzy Volterra-Fredholm integral equations ...

Similarly, we can construct a homotopy when consider, $N[\bar{u}]=0$, as follows:

$(1-q) L\left[\bar{\phi}(x ; q, r)-\bar{u}_{0}(x, r)\right]-q h H_{1}(x) N[\phi(x ; q, r)]=\hat{H}\left[\phi(x ; q, r) ; \bar{u}_{0}(x, r), H_{1}(x), h, q\right]$.

It should be emphasized that we have great freedom to choose the initial guess $\bar{u}_{0}(x, r)$, the auxiliary linear operator $L$, the non-zero auxiliary parameter $h$, and the auxiliary function $H_{1}(x)$. Enforcing the homotopy Eq.(39) to be zero, i.e.,

$$
\hat{H}_{1}\left[\bar{\phi}(x ; q, r) ; \bar{u}_{0}(x, r), H_{1}(x), h, q\right]=0,
$$

we have the so-called zero-order deformation equation

$$
(1-q) L\left[\bar{\phi}(x ; q, r)-\bar{u}_{0}(x, r)\right]=q h H_{1}(x) N[\phi(x ; q, r)],
$$

when $q=0$, the zero-order deformation Eq.(41) becomes

$$
\bar{\phi}(x ; 0, r)=\bar{u}_{0}(x, r),
$$

and when $q=1$, since $h \neq 0$ and $H_{1}(x) \neq 0$, the zero-order deformation Eq.(41) is equivalent to

$$
\bar{\phi}(x ; 1, r)=\bar{u}(x, r) .
$$

Thus, according to Eqs.(42) and (43), as the embedding parameter $q$ increases from 0 to $1, \phi(x ; q, r)$ varies continuously from the initial approximation $\bar{u}_{0}(x, r)$ to the exact solution $\bar{u}(x, r)$. Such a kind of continuous variation is called deformation in homotopy. Due to Taylor's theorem, $\phi(x ; q, r)$ can be expanded in a power series of $q$ as follows

$$
\bar{\phi}(x ; q, r)=\bar{u}_{0}(x, r)+\sum_{m=1}^{\infty} \bar{u}_{m}(x, r) q^{m},
$$

where,

$$
\bar{u}_{m}(x, r)=\left.\frac{1}{m !} \frac{\partial^{m} \bar{\phi}(x ; q, r)}{\partial q^{m}}\right|_{q=0} .
$$

Let the initial guess $\bar{u}_{0}(x, r)$, the auxiliary linear parameter $L$, the nonzero auxiliary parameter $h$ and the auxiliary function $H_{1}(x)$ be properly chosen so that the power series Eq.(44) of $\bar{\phi}(x ; q, r)$ converges at $q=1$, then, we have under these assumptions the solution series

$$
\bar{u}(x, r)=\bar{\phi}(x ; 1, r)=\bar{u}_{0}(x, r)+\sum_{m=1}^{\infty} \bar{u}_{m}(x, r) .
$$


A.A. Hamoud, K.P. Ghadle - Fuzzy Volterra-Fredholm integral equations ...

From Eq.(44), we can write Eq.(41) as follows:

$$
\begin{aligned}
(1-q) L\left[\bar{\phi}(x ; q, r)-\bar{u}_{0}(x, r)\right] & =(1-q) L\left[\sum_{m=1}^{\infty} \bar{u}_{m}(x, r) q^{m}\right] \\
& =q h H_{1}(x) N[\bar{\phi}(x ; q, r)],
\end{aligned}
$$

then,

$$
L\left[\sum_{m=1}^{\infty} \bar{u}_{m}(x, r) q^{m}\right]-q L\left[\sum_{m=1}^{\infty} \bar{u}_{m}(x, r) q^{m}\right]=q h H_{1}(x) N[\bar{\phi}(x ; q, r)] .
$$

By differentiating Eq.(47) $m$-times with respect to $q$, we obtain

$$
\begin{aligned}
\left\{L\left[\sum_{m=1}^{\infty} \bar{u}_{m}(x, r) q^{m}\right]-q L\left[\sum_{m=1}^{\infty} \bar{u}_{m}(x, r) q^{m}\right]\right\}^{(m)} & =q h H_{1}(x) N[\bar{\phi}(x ; q, r)]^{(m)} \\
& =m ! L\left[\bar{u}_{m}(x)-\bar{u}_{m-1}(x, r)\right] \\
& =\left.h H_{1}(x) m \frac{\partial^{m-1} N[\bar{\phi}(x ; q, r)]}{\partial q^{m-1}}\right|_{q=0} .
\end{aligned}
$$

Therefore,

$$
L\left[\bar{u}_{m}(x, r)-\chi_{m} \bar{u}_{m-1}(x, r)\right]=h H_{1}(x) \Re_{m}\left(\bar{u}_{m-1}(x, r)\right),
$$

where,

$$
\Re_{m}\left(\bar{u}_{m-1}(x)\right)=\left.\frac{1}{(m-1) !} \frac{\partial^{m-1} N[\varphi(x ; q)]}{\partial q^{m-1}}\right|_{q=0}
$$

and

$$
\chi_{m}= \begin{cases}0 & m \leq 1 \\ 1 & m>1\end{cases}
$$

Note that the high-order deformation Eq.(49) is governing the linear operator $L$, and the term $\Re_{m}\left(\bar{u}_{m-1}(x, r)\right)$ can be expressed simply by Eq.(50) for any nonlinear operator $N$.

To obtain the approximation solution of Eq.(8), according to HAM, let us do

$$
N[\bar{u}(x, r)]=\bar{u}(x, r)-\bar{f}(x, r)-\mu_{1} \int_{a}^{x} \overline{K_{1}(x, t) G_{1}(t, u(t, r))} d t-\mu_{2} \int_{a}^{b} \overline{K_{2}(x, t) G_{2}(t, u(t, r))} d t
$$


so,

$$
\begin{array}{r}
\Re_{m}\left(\bar{u}_{m-1}(x, r)\right)=\bar{u}_{m-1}(x, r)-\bar{f}(x, r)-\mu_{1} \int_{a}^{x} \overline{K_{1}(x, t) G_{1}(t, u(t, r))} d t \\
-\mu_{2} \int_{a}^{b} \overline{K_{2}(x, t) G_{2}(t, u(t, r))} d t-\left(1-\chi_{m}\right) \bar{f}(x, r), m \geq 1 .
\end{array}
$$

Substituting Eq.(51) into Eq. (49)

$$
\begin{aligned}
L\left[\bar{u}_{m}(x, r)-\chi_{m} \bar{u}_{m-1}(x, r)\right]= & h H_{1}(x)\left[\bar{u}_{m-1}(x, r)-\mu_{1} \int_{a}^{x} \overline{K_{1}(x, t) G_{1}(t, u(t, r))} d t\right. \\
& \left.-\mu_{2} \int_{a}^{b} \overline{K_{2}(x, t) G_{2}(t, u(t, r))} d t-\left(1-\chi_{m}\right) \bar{f}(x, r)\right] .
\end{aligned}
$$

We take an initial guess $\bar{u}_{0}(x, r)=\bar{f}(x, r)$, an auxiliary linear operator $L \bar{u}=\bar{u}$, a nonzero auxiliary parameter $h=-1$, and auxiliary function $H_{1}(x)=1$. This is substituted into Eq.(52) to give the recurrence relation

$$
\begin{aligned}
\bar{u}_{0}(x, r) & =\bar{f}(x, r) \\
\bar{u}_{n+1}(x, r) & =\mu_{1} \int_{a}^{x} \overline{K_{1}(x, t) G_{1}\left(t, u_{n}(t, r)\right)} d t+\mu_{2} \int_{a}^{b} \overline{K_{2}(x, t) G_{2}\left(t, u_{n}(t, r)\right)} d t, n \geq 0
\end{aligned}
$$

From Eqs.(38), and Eqs.(53) we approximate $\tilde{u}(x, r)=[\underline{u}(x, r), \bar{u}(x, r)]$ by

$$
\underline{u}(x, r)=\lim _{n \rightarrow \infty} \underline{u}_{n}, \quad \bar{u}(x, r)=\lim _{n \rightarrow \infty} \bar{u}_{n} .
$$

\section{Main Results}

In what follows we will prove the uniqueness of the solution and convergence of the methods by using the following assumptions.

(A1) There exist two constants $M_{1}, M_{2}>0$ such that,

$$
\left|\mu_{1} K_{1}(x, t)\right| \leq M_{1},\left|\mu_{2} K_{2}(x, t)\right| \leq M_{2}, \quad \forall a \leq x, t \leq b .
$$

(A2) Suppose the nonlinear operators $G_{1}(t, \tilde{u}(t)), G_{2}(t, \tilde{u}(t))$ are satisfied in Lipschitz conditions with

$$
\begin{aligned}
\left|G_{1}(t, \tilde{u}(t))-G_{1}\left(t, \tilde{u}^{*}(t)\right)\right| & \leq L_{1}\left|\tilde{u}-\tilde{u}^{*}\right| \\
\left|G_{2}(t, \tilde{u}(t))-G_{2}\left(t, \tilde{u}^{*}(t)\right)\right| & \leq L_{2}\left|\tilde{u}-\tilde{u}^{*}\right| .
\end{aligned}
$$


(A3) Consider $\tilde{f}(x)$ is bounded $\forall x \in[a, b]$.

Theorem 2. Assume that (A1), (A2) and (A3) hold. Then Eq.(6) has a unique solution, if $0<\alpha<1$, where

$$
\alpha=\left(M_{1} L_{1}+M_{2} L_{2}\right)(b-a) .
$$

Proof. Let $\tilde{u}$ and $\tilde{u}^{*}$ be two different solutions of Eq.(6) then

$$
\begin{aligned}
\left|\tilde{u}-\tilde{u}^{*}\right|= & \mid \tilde{f}(x)+\mu_{1} \int_{a}^{x} K_{1}(x, t) G_{1}(t, \tilde{u}(t)) d t+\mu_{2} \int_{a}^{b} K_{2}(x, t) G_{2}(t, \tilde{u}(t)) d t \\
& -\left(\tilde{f}(x)+\mu_{1} \int_{a}^{x} K_{1}(x, t) G_{1}\left(t, \tilde{u}^{*}(t)\right) d t+\mu_{2} \int_{a}^{b} K_{2}(x, t) G_{2}\left(t, \tilde{u}^{*}(t)\right) d t\right) \mid, \\
= & \mid \mu_{1} \int_{a}^{x} K_{1}(x, t) G_{1}(t, \tilde{u}(t)) d t+\mu_{2} \int_{a}^{b} K_{2}(x, t) G_{2}(t, \tilde{u}(t)) d t \\
& -\left(\mu_{1} \int_{a}^{x} K_{1}(x, t) G_{1}\left(t, \tilde{u}^{*}(t)\right) d t+\mu_{2} \int_{a}^{b} K_{2}(x, t) G_{2}\left(t, \tilde{u}^{*}(t)\right) d t\right) \mid, \\
= & \mid \int_{a}^{x} \mu_{1} K_{1}(x, t) G_{1}(t, \tilde{u}(t)) d t-\int_{a}^{x} \mu_{1} K_{1}(x, t) G_{1}\left(t, \tilde{u}^{*}(t)\right) d t \\
& +\int_{a}^{b} \mu_{2} K_{2}(x, t) G_{2}(t, \tilde{u}(t)) d t-\int_{a}^{b} \mu_{2} K_{2}(x, t) G_{2}\left(t, \tilde{u}^{*}(t)\right) d t \mid \\
= & \mid \int_{a}^{x} \mu_{1} K_{1}(x, t)\left[G_{1}(t, \tilde{u}(t))-G_{1}\left(t, \tilde{u}^{*}(t)\right)\right] d t \\
& +\int_{a}^{b} \mu_{2} K_{2}(x, t)\left[G_{2}(t, \tilde{u}(t))-G_{2}\left(t, \tilde{u}^{*}(t)\right)\right] d t \mid \\
& \\
& \quad \leq \int_{a}^{x}\left|\mu_{1} K_{1}(x, t)\right|\left|G_{1}(t, \tilde{u}(t))-G_{1}\left(t, \tilde{u}^{*}(t)\right)\right| d t \\
& +\int_{a}^{b}\left|\mu_{2} K_{2}(x, t)\right|\left|G_{2}(t, \tilde{u}(t))-G_{2}\left(t, \tilde{u}^{*}(t)\right)\right| d t \\
\leq & M_{1} L_{1}(b-a)\left|\tilde{u}-\tilde{u}^{*}\right|+M_{2} L_{2}(b-a)\left|\tilde{u}-\tilde{u}^{*}\right| \\
& \left(M_{1} L_{1}+M_{2} L_{2}\right)(b-a)\left|\tilde{u}-\tilde{u}^{*}\right| \\
& \alpha\left|\tilde{u}-\tilde{u}^{*}\right|,
\end{aligned}
$$

from which we get $(1-\alpha)\left|\tilde{u}-\tilde{u}^{*}\right| \leq 0$. Since $0<\alpha<1$, then $\left|\tilde{u}-\tilde{u}^{*}\right|=0$ implies $\tilde{u}=\tilde{u}^{*}$ and this completes the proof.

Similarly, we can prove the uniqueness of the solution of Eq.(5). 
Theorem 3. If the series solution $\tilde{u}(x, r)=\sum_{i=0}^{\infty} \tilde{u}_{i}(x, r)$ obtained by the using $M A D M$ is convergent, then it converges to the exact solution of the Eq.(6) when $0<\alpha<1$ and $\left\|\tilde{u}_{1}(x, r)\right\|<\infty$.

Proof. Denote as $(C[a, b],\|\cdot\|)$ the Banach space of all continuous functions on $J=$ $[a, b]$ with $\left|\tilde{u}_{1}(x, r)\right| \leq \infty$ for all $x$ in $J$.

Firstly, we define the sequence of partial sums $\underline{s}_{n}$, let $\underline{s}_{n}$ and $\underline{s}_{m}$ be arbitrary partial sums with $n \geq m$. We are going to prove that $\underline{s}_{n}=\sum_{i=0}^{n} \underline{u}_{i}(x, r)$ is a Cauchy sequence in this Banach space:

$$
\begin{aligned}
\left\|\underline{s}_{n}-\underline{s}_{m}\right\| & =\max _{\forall x \in J}\left|\underline{s}_{n}-\underline{s}_{m}\right| \\
& =\max _{\forall x \in J}\left|\sum_{i=0}^{n} \underline{u}_{i}(x, r)-\sum_{i=0}^{m} \underline{u}_{i}(x, r)\right| \\
& =\max _{\forall x \in J}\left|\sum_{i=m+1}^{n} \underline{u}_{i}(x, r)\right| \\
& =\max _{\forall x \in J}\left|\sum_{i=m+1}^{n}\left[\mu_{1} \int_{a}^{x} K_{1}(x, t) \underline{A}_{i}(t) d t+\mu_{2} \int_{a}^{b} K_{2}(x, t) \underline{B}_{i}(t) d t\right]\right| \\
& \left.=\max _{\forall x \in J} \mid \int_{a}^{x} \mu_{1} K_{1}(x, t) \sum_{i=m}^{n-1} \underline{A}_{i}(t) d t+\int_{a}^{b} \mu_{2} K_{2}(x, t) \sum_{i=m}^{n-1} \underline{B}_{i}(t) d t\right) \mid .
\end{aligned}
$$

From [12], we have

$$
\begin{aligned}
& \sum_{i=m}^{n-1} \underline{A}_{i}=G_{1}\left(t, \underline{s}_{n-1}\right)-G_{1}\left(t, \underline{s}_{m-1}\right), \\
& \sum_{i=m}^{n-1} \underline{B}_{i}=G_{2}\left(t, \underline{s}_{n-1}\right)-G_{2}\left(t, \underline{s}_{m-1}\right) .
\end{aligned}
$$


So,

$$
\begin{aligned}
\left\|\underline{s}_{n}-\underline{s}_{m}\right\|= & \max _{\forall x \in J} \mid \int_{a}^{x} \mu_{1} K_{1}(x, t)\left(G_{1}\left(t, \underline{s}_{n-1}\right)-G_{1}\left(t, \underline{s}_{m-1}\right)\right) d t \\
& +\int_{a}^{b} \mu_{2} K_{2}(x, t)\left(G_{2}\left(t, \underline{s}_{n-1}\right)-G_{2}\left(t, \underline{s}_{m-1}\right)\right) d t \mid \\
\leq & \max _{\forall x \in J}\left(\int_{a}^{x}\left|\mu_{1} K_{1}(x, t) \|\left(G_{1}\left(t, \underline{s}_{n-1}\right)-G_{1}\left(t, \underline{s}_{m-1}\right)\right)\right| d t\right. \\
& \left.+\int_{a}^{b}\left|\mu_{2} K_{2}(x, t) \|\left(G_{2}\left(t, \underline{s}_{n-1}\right)-G_{2}\left(t, \underline{s}_{m-1}\right)\right)\right| d t\right) \\
\leq & M_{1} L_{1}\left\|\underline{s}_{n-1}-\underline{s}_{m-1}\right\|(b-a)+M_{2} L_{2}\left\|\underline{s}_{n-1}-\underline{s}_{m-1}\right\|(b-a) \\
= & \left(L_{1} M_{1}+M_{2} L_{2}\right)(b-a)\left\|\underline{s}_{n-1}-\underline{s}_{m-1}\right\| \\
= & \alpha\left\|\underline{s}_{n-1}-\underline{s}_{m-1}\right\| .
\end{aligned}
$$

Let $n=m+1$, then

$$
\left\|\underline{s}_{n}-\underline{s}_{m}\right\| \leq \alpha\left\|\underline{s}_{m}-\underline{s}_{m-1}\right\| \leq \alpha^{2}\left\|\underline{s}_{m-1}-\underline{s}_{m-2}\right\| \leq \cdots \leq \alpha^{m}\left\|\underline{s}_{1}-\underline{s}_{0}\right\|
$$

. So,

$$
\begin{aligned}
\left\|\underline{s}_{n}-\underline{s}_{m}\right\| & \leq\left\|\underline{s}_{n}-\underline{s}_{m}\right\| \leq\left\|\underline{s}_{m+1}-\underline{s}_{m}\right\|+\left\|\underline{s}_{m+2}-\underline{s}_{m+1}\right\|+\cdots+\left\|\underline{s}_{n}-\underline{s}_{n-1}\right\| \\
& \leq\left[\alpha^{m}+\alpha^{m+1}+\cdots+\alpha^{n-1}\right]\left\|\underline{s}_{1}-\underline{s}_{0}\right\| \\
& \leq \alpha^{m}\left[1+\alpha+\alpha^{2}+\cdots+\alpha^{n-m-1}\right]\left\|\underline{s}_{1}-\underline{s}_{0}\right\| \\
& \leq \alpha^{m}\left(\frac{1-\alpha^{n-m}}{1-\alpha}\right)\left\|\underline{u}_{1}(x, r)\right\| .
\end{aligned}
$$

Since $0<\alpha<1$, we have $\left(1-\alpha^{n-m}\right)<1$, then

$$
\left\|\underline{s}_{n}-\underline{s}_{m}\right\| \leq \frac{\alpha^{m}}{1-\alpha}\left\|\underline{u}_{1}(x, r)\right\| .
$$

But $\left|\underline{u}_{1}(x, r)\right|<\infty$, so, as $m \longrightarrow \infty$, then $\left\|\underline{s}_{n}-\underline{s}_{m}\right\| \longrightarrow 0$. We conclude that $\underline{s}_{n}$ is a Cauchy sequence in $C[J]$, therefore

$$
\underline{u}(x, r)=\lim _{n \rightarrow \infty} \underline{u}_{n}(x, r) .
$$

Similarly, we have $\bar{s}_{n}$ is cauchy sequence, then, we can write

$$
\bar{u}(x, r)=\lim _{n \rightarrow \infty} \bar{u}_{n}(x, r) .
$$


Therefore,

$$
\tilde{u}(x, r)=\lim _{n \rightarrow \infty} \tilde{u}_{n}(x, r)
$$

the series is convergence and the proof is complete.

Theorem 4. If the solution $\tilde{u}(x)=\lim _{n \rightarrow \infty} \tilde{u}_{n}(x)$ obtained by using VIM is convergent, then it converges to the exact solution of the Eq.(6) with $0<\alpha<1$.

Proof. The iteration formula as follows:

$$
\begin{aligned}
\underline{u}_{n+1}(x, r)= & \underline{u}_{n}(x, r)-\int_{0}^{x}\left[\underline{u}_{n}(s, r)-\underline{f}(s, r)-\mu_{1} \int_{a}^{s} K_{1}(s, t) G_{1}\left(t, \underline{u}_{n}(t, r)\right) d t\right. \\
& \left.-\mu_{2} \int_{a}^{b} K_{2}(s, t) G_{2}\left(t, \underline{u}_{n}(t, r)\right) d t\right] d s
\end{aligned}
$$

we can write

$$
\begin{aligned}
\underline{u}(x, r)= & \underline{u}(x, r)-\int_{0}^{x}\left[\underline{u}(s, r)-\underline{f}(s, r)-\mu_{1} \int_{a}^{s} K_{1}(s, t) G_{1}(t, \underline{u}(t, r)) d t\right. \\
& \left.-\mu_{2} \int_{a}^{b} K_{2}(s, t) G_{2}(t, \underline{u}(t, r)) d t\right] d s .
\end{aligned}
$$

By subtracting Eq.(55) from Eq.(56),

$$
\begin{aligned}
\underline{u}_{n+1}(x, r)-\underline{u}(x, r)= & \underline{u}_{n}(x, r)-\underline{u}(x, r)-\int_{0}^{x}\left[\underline{u}_{n}(s, r)-\underline{u}(s, r)\right. \\
& -\mu_{1} \int_{a}^{s} K_{1}(s, t)\left[G_{1}\left(t, \underline{u}_{n}(t, r)\right)-G_{1}(t, \underline{u}(t, r))\right] d t \\
& \left.-\mu_{2} \int_{a}^{b} K_{2}(s, t)\left[G_{2}\left(t, \underline{u}_{n}(t, r)\right)-G_{2}(t, \underline{u}(t, r))\right] d t\right] d s .
\end{aligned}
$$

If we set, $\underline{e}_{n+1}(x, r)=\underline{u}_{n+1}(x, r)-\underline{u}(x, r)$, and $\underline{e}_{n}(x, r)=\underline{u}_{n}(x, r)-\underline{u}(x, r)$ then

$$
\begin{aligned}
\underline{e}_{n+1}(x, r)= & \underline{e}_{n}(x, r)-\int_{0}^{x}\left[\underline{e}_{n}(s, r)-\mu_{1} \int_{a}^{s} K_{1}(s, t)\left[G_{1}\left(t, \underline{u}_{n}(t, r)\right)-G_{1}(t, \underline{u}(t, r))\right] d t\right. \\
& \left.-\mu_{2} \int_{a}^{b} K_{2}(s, t)\left[G_{2}\left(t, \underline{u}_{n}(t, r)\right)-G_{2}(t, \underline{u}(t, r))\right] d t\right] d s \\
& +\underline{e}_{n}(x, r)-\underline{e}_{n}(x, 0) \\
\leq & \underline{e}_{n}(x, r)\left(1-(b-a)\left(M_{1} L_{1}+M_{2} L_{2}\right)\right) \\
= & (1-\alpha) \underline{e}_{n}(x, r),
\end{aligned}
$$


therefore,

$$
\begin{aligned}
\left\|\underline{e}_{n+1}\right\| & =\max _{\forall x \in J}\left|\underline{e}_{n+1}\right| \\
& \leq(1-\alpha) \max _{\forall x \in J}\left|\underline{e}_{n}\right| \\
& =\left\|\underline{e}_{n}\right\|,
\end{aligned}
$$

since $0<\alpha<1$, then $\left\|\underline{e}_{n}\right\| \longrightarrow 0$. Similarly, we can proof the procedure of $\bar{u}(x, t)$. So, the series converges and the proof is complete.

Theorem 5. If the series solution $\underline{u}(x, t)=\sum_{m=0}^{\infty} \underline{u}_{m}(x, t)$ obtained by the $m$ order deformation is convergent, then it converges to the exact solution of the fuzzy Volterra-Fredholm integral equation (7).

Proof. We assume $\sum_{m=0}^{\infty} \underline{u}_{m}(x, t)$ converge uniformly to $\underline{u}(x, t)$

$$
\underline{u}(x, r)=\sum_{m=0}^{\infty} \underline{u}_{m}(x, r),
$$

where,

$$
\lim _{m \rightarrow \infty} \underline{u}_{m}(x, r)=0
$$

We can write,

$$
\begin{aligned}
\sum_{m=1}^{n}\left[\underline{u}_{m}(x, r)-\chi_{m} \underline{u}_{m-1}(x, r)\right]= & \underline{u}_{1}(x, r)+\left(\underline{u}_{2}(x, r)-\underline{u}_{1}(x, r)\right)+\left(\underline{u}_{3}(x, r)\right. \\
& \left.-\tilde{u}_{2}(x, r)\right)+\cdots+\left(\underline{u}_{n}(x, r)-\underline{u}_{n-1}(x, r)\right) \\
= & \underline{u}_{n}(x, r) .
\end{aligned}
$$

Hence, from Eq.(59)

$$
\lim _{n \rightarrow \infty} \underline{u}_{n}(x, r)=0
$$

So, using Eq.(60) and the definition of the linear operator $L$, we have

$$
\sum_{m=1}^{\infty} L\left[\underline{u}_{m}(x, r)-\chi_{m} \underline{u}_{m-1}(x, r)\right]=L \sum_{m=1}^{\infty}\left[\underline{u}_{m}(x, r)-\chi_{m} \underline{u}_{m-1}(x, r)\right]=0 .
$$

Therefore from Eq.(60), we can obtain that

$$
\sum_{m=1}^{\infty} L\left[\underline{u}_{m}(x, r)-\chi_{m} \underline{u}_{m-1}(x, r)\right]=h H(x) \sum_{m=1}^{\infty} \Re_{m-1}\left(\underline{u}_{m-1}(x, r)\right)=0 .
$$


Since $h \neq 0$ and $H(x) \neq 0$, we have

$$
\sum_{m=1}^{\infty} \Re_{m-1}\left(\underline{u}_{m-1}(x, r)\right)=0 .
$$

By substituting $\Re_{m-1}\left(\underline{u}_{m-1}(x, r)\right)$ into the relation (61) and simplifying it, we have

$$
\begin{aligned}
\Re_{m-1}\left(\underline{u}_{m-1}(x, r)\right) & =\sum_{m=1}^{\infty}\left[\underline{u}_{m-1}(x, r)-\mu_{1} \int_{a}^{x} K_{1}(x, t) G_{1}\left(t, \underline{u}_{m-1}(t, r)\right) d t\right. \\
& \left.-\mu_{2} \int_{a}^{b} K_{2}(x, t) G_{2}\left(t, \underline{u}_{m-1}(t, r)\right) d t-\left(1-\chi_{m}\right) \underline{f}(x, r)\right] \\
& =\underline{u}(x, r)(x)-\tilde{f}(x, r)-\mu_{1} \int_{a}^{x} K_{1}(x, t)\left[\sum_{m=1}^{\infty} G_{1}\left(t, \underline{u}_{m-1}(t, r)\right)\right] d t \\
& -\mu_{2} \int_{a}^{b} K_{2}(x, t)\left[\sum_{m=1}^{\infty} G_{2}\left(t, \underline{u}_{m-1}(t, r)\right)\right] d t .
\end{aligned}
$$

From Eq.(61) and Eq.(62), we have

$$
\underline{u}(x, r)=\underline{f}(x, r)+\mu_{1} \int_{a}^{x} K_{1}(x, t) G_{1}(t, \underline{u}(t, r)) d t+\mu_{2} \int_{a}^{b} K_{2}(x, t) G_{2}(t, \underline{u}(t, r)) d t,
$$

therefore, $\underline{u}(x, r)$ must be the exact solution of Eq.(7).

Similarly, we can proof the procedure of $\bar{u}(x, t)$. Then, $\tilde{u}(x)$ must be the exact solution of Eq.(6), and the proof is complete.

\section{Numerical EXAMPles}

In this section, we solve the fuzzy Volterra-Fredholm integral equation of the first and second kind by using the ADM, MADM, VIM and HAM. The program has been provided with Mathematica 6.

Algorithm :

Step 1. Set $n \longleftarrow 0$,

Step 2. Solve the systems (5) or (6),

Step 3. If $\left|\tilde{u}_{n+1}-\tilde{u}_{n}\right|<\epsilon$ then go to step 4, else $n \longleftarrow n+1$ and go to step 2 .

Step 4. Print $\tilde{u}_{n}$ as the approximate solution. 
A.A. Hamoud, K.P. Ghadle - Fuzzy Volterra-Fredholm integral equations ...

\section{Example 7.1}

Consider the fuzzy Volterra-Fredholm integral equation of the first kind as follows:

$$
\tilde{f}(x)=\int_{0}^{x}\left(x^{2}+2 t\right) \tilde{u}(t) d t+\int_{0}^{0.8} \sqrt{x+t} \tilde{u}(t) d t
$$

whear,

$$
\begin{aligned}
& \underline{f}(x, r)=r x+\frac{1}{8}-\frac{1}{8} r-\frac{4}{17} x^{2}+\frac{4}{17} x^{2} r, \\
& \bar{f}(x, r)=2 r-r x+\frac{1}{8} r-\frac{1}{8}+\frac{4}{17} x^{2} r-\frac{4}{17} x^{2},
\end{aligned}
$$

and,

$$
r=0.25, \quad \epsilon=10^{-4} .
$$

We reduce Eq.(63) of the first kind to the second kind by differentiating one time with respect to $x$ we get:

$$
\begin{gathered}
\tilde{f}^{\prime}(x)=\left(x^{2}+2 x\right) \tilde{u}(x)+\int_{0}^{x}(2 x+2 t) \tilde{u}(t) d t+\int_{0}^{0.8} \frac{1}{2 \sqrt{x+t}} \tilde{u}(t) d t \\
\tilde{u}(x)=\frac{\tilde{f}^{\prime}(x)}{\left(x^{2}+2 x\right)}-2 \int_{0}^{x} \frac{(x+t)}{\left(x^{2}+2 x\right)} \tilde{u}(t) d t-\frac{1}{2} \int_{0}^{0.8} \frac{\tilde{u}(t)}{\left(x^{2}+2 x\right) \sqrt{x+t}} d t .
\end{gathered}
$$

\begin{tabular}{lcccc}
\hline $\mathrm{x}$ & $\operatorname{ADM}_{(n=9)}$ & $M A D M_{(n=6)}$ & $V I M_{(n=4)}$ & $H A M_{(n=4)}$ \\
\hline 0.1 & 0.4039474 & 0.4724382 & 0.4959472 & 0.4978465 \\
0.2 & 0.4138278 & 0.4827677 & 0.4967593 & 0.4989678 \\
0.3 & 0.4329266 & 0.5035533 & 0.5089546 & 0.5099838 \\
0.4 & 0.4536241 & 0.5258682 & 0.5309465 & 0.5327889 \\
0.5 & 0.4662346 & 0.5367692 & 0.5339489 & 0.5476479 \\
0.6 & 0.4845248 & 0.5547509 & 0.5564829 & 0.5754394 \\
\hline
\end{tabular}

Table 1: The Obtained Solutions for Example 7.1

\section{Example 7.2}

Consider the fuzzy Volterra-Fredholm integral equation of the second kind as follows:

$$
\tilde{u}(x)=\tilde{f}(x)+\int_{0}^{x} \sin (x) \sin \left(\frac{t}{2}\right) \tilde{u}^{3}(t) d t+\int_{0}^{0.6} \sin \left(\frac{x}{2}\right) \sin (t)\left(1+\tilde{u}^{2}(t)\right) d t,
$$


A.A. Hamoud, K.P. Ghadle - Fuzzy Volterra-Fredholm integral equations ...

whear,

$$
\begin{aligned}
& \underline{f}(x, r)=\sin \left(\frac{x}{2}\right)\left(\frac{13}{15}\left(r^{2}+r\right)+\frac{2}{15}\left(4-r^{3}-r\right),\right. \\
& \bar{f}(x, r)=\sin \left(\frac{x}{2}\right)\left(\frac{2}{15}\left(r^{2}+r\right)+\frac{13}{15}\left(4-r^{3}-r\right),\right.
\end{aligned}
$$

and,

$$
r=0.3, \quad \epsilon=10^{-2}, \quad 0 \leq x, t \leq 0.6
$$

\begin{tabular}{lcccc}
\hline $\mathrm{x}$ & $A D M_{(n=11)}$ & $M A D M_{(n=7)}$ & $V I M_{(n=4)}$ & $H A M_{(n=4)}$ \\
\hline 0.1 & 0.2203548375 & 0.220364689 & 0.220466127 & 0.2204663982 \\
0.2 & 0.3062332542 & 0.306447853 & 0.306329751 & 0.3063488741 \\
0.3 & 0.4035946723 & 0.403687924 & 0.403659665 & 0.4037996457 \\
0.4 & 0.5233741235 & 0.523298269 & 0.523379658 & 0.5234862764 \\
0.5 & 0.5964831157 & 0.596683295 & 0.614656263 & 0.6259432736 \\
0.6 & 0.6523678927 & 0.652287496 & 0.652356871 & 0.6524855123 \\
\hline
\end{tabular}

Table 2: The Obtained Solutions for Example 7.2

The above tables show comparison between the approximate solutions by using ADM, MDM, VIM, and HAM for results of the examples 7.1 and 7.2.

\section{Discussion the Results and Comparison Among the Four Methods}

The comparison among of the methods, it can be seen from the results of the above examples:

- The four methods are powerful, efficient and give approximations of higher accuracy. Also, they can produce closed-form solutions if they exist.

- Although the results obtained by these methods when applied to fuzzy VolterraFredholm integral equations are the same approximately. HAM is seen to be much easier and more convenient than the others.

- The VIM or HAM have many merits and more advantages over other methods. They can be introduced to overcome the difficulties arising in calculating Adomian polynomials. VIM and HAM reduce the volume of calculations by requiring Adomian polynomials. The iterations methods are direct and straightforward. 
A.A. Hamoud, K.P. Ghadle - Fuzzy Volterra-Fredholm integral equations ...

- HAM handles fuzzy Volterra-Fredholm integral equations in a simple manner by deforming a difficult problem into a simple one.

- One advantage of VIM is that the initial solution can be freely chosen with some unknown parameters. An interesting point about this method is that with few number of iterations, or even in some cases with only one iteration, it can produce a very accurate approximate solution.

- The VIM has a more rapid convergence than the MDM. Also, the number of computations in VIM is less than the ones in MDM.

\section{Conclusion}

We discussed four different methods for solving fuzzy Volterra-Fredholm integral equations, namely, Adomian decomposition method, Modified decomposition method, variational iteration method and Homotopy analysis method. To assess the accuracy of each method, the test examples with the known exact solution is used. The study outlines important features of these methods as well as sheds some light on advantages of one method over the other. The results show that these methods are very efficient, convenient and can be adapted to fit a larger class of problems. The comparison reveals that although the numerical results of these methods are similar approximately, HAM is the easiest, the most efficient and convenient.

\section{REFERENCES}

[1] K. Abbaoui, Y. Cherruault, Convergence of Adomian's method applied to nonlinear equations, Math. Comput. Model, 20 (1994), 69-73.

[2] G. Adomian, A review of the decomposition method in applied mathematics, J. Math. Anal. Appl. (1988), 501-544.

[3] T. Allahviranloo, The Adomian decomposition method for fuzzy system of linear equations, J. Appl. Math. Comput. 163 (2005), 553-563.

[4] M. Araghi, S. Behzadi, Solving nonlinear Volterra-Fredholm integro-differential equations using the modified Adomian decomposition method, Comput. Methods in Appl. Math. 9 (2009), 1-11.

[5] M. Al-Smadi, G. Gumah, On the homotopy analysis method for fractional SEIR epidemic model, Research Journal of Applied Sciences, Engineering and Technology, 18, 7 (2014), 3809-3820.

[6] N. Bildik, M. Inc, Modified decomposition method for nonlinear VolterraFredholm integral equations, Chaos Solitons Fractals, 33 (2007), 308-313. 
A.A. Hamoud, K.P. Ghadle - Fuzzy Volterra-Fredholm integral equations ...

[7] E. Babolian, J. Biazar, A. Vahidi, Solution of a system of nonlinear equations by Adomian decomposition method, Appl. Math. Comput. 150 (2004), 847-854.

[8] E. Babolian, A. Davari, Numerical implementation of Adomian decomposition method, Appl. Math. Comput. (2004), 301-305.

[9] E. Babolian, H. Goghary, S. Abbasbandy, Numerical solution of linear Fredholm fuzzy integral equations of the second kind by Adomian method, Appl. Math. Comput. 161 (2005), 733-744.

[10] M. Bani Issa, A. Hamoud, K. Ghadle and Giniswamy, Hybrid method for solving nonlinear Volterra-Fredholm integro-differential equations, J. Math. Comput. Sci. 7, (4) (2017), 625-641.

[11] B. Bede, S. Gal, Generalizations of the differentiability of fuzzy number-valued functions with applications to fuzzy differential equations, Fuzzy Sets and Systems, 151 (2005), 581-599.

[12] Y. Chalco-Cano, H. Roman-Flores, On new solutions of fuzzy differential equations, Chaos Solitons Fractals, 38 (2008), 112-119.

[13] S. Chang, L. Zadeh, On fuzzy mapping and control, IEEE Trans. Systems, Man cybernet, 2 (1972), 30-34.

[14] D. Dubois, H. Prade, Towards fuzzy differential calculus. I: integration of fuzzy mappings, Fuzzy Sets and Systems, 8 (1982), 1-17.

[15] D. Dubois, H. Prade, Operations on fuzzy numbers, Int. J. systems Science, 9 (1978), 613-626.

[16] I. El-Kalaa, Convergence of the Adomian method applied to a class of nonlinear integral equations, App. Math. Comput. 21 (2008), 327-376.

[17] M. Friedman, M. Ming, A. Kandel, Numerical solution of fuzzy differential and integral equations, Fuzzy Sets and System, 106 (1999), 35-48.

[18] R. Goetschel, W. Voxman, Elementary calculus, Fuzzy Sets and Systems, 18 (1986), 31-43.

[19] O. Kaleva, Fuzzy differential equation, Fuzzy Sets and Systems, 24 (1987), 301317.

[20] A. Hamoud, K. Ghadle, On the numerical solution of nonlinear VolterraFredholm integral equations by variational iteration method, Int. J. Adv. Sci. Res. 3 (2016), 45-51.

[21] A. Hamoud, K. Ghadle, Study of the approximate solution of fuzzy VolterraFredholm integral equations by using (ADM). Elixir Appl. Math. 98 (2016), 4256742573.

[22] A. Hamoud, K. Ghadle, The Combined modified Laplace with Adomian decomposition method for solving the nonlinear Volterra-Fredholm integro-differential equations, J. Korean Soc. Ind. Appl. Math. 21 (2017), 17-28. 
A.A. Hamoud, K.P. Ghadle - Fuzzy Volterra-Fredholm integral equations ...

[23] A. Hamoud, K. Ghadle, The reliable modified of Laplace Adomian decomposition method to solve nonlinear interval Volterra-Fredholm integral equations, Korean J. Math. 25 (2017), 323-334.

[24] A. Hamoud and K. Ghadle, Modified Adomian decomposition method for solving fuzzy Volterra-Fredholm integral equations, J. Indian Math. Soc. 85(1-2) (2018), 5269.

[25] A. Hamoud and K. Ghadle, Existence and uniqueness results for fractional Volterra-Fredholm integro-differential equations, Int. J. Open Problems Compt. Math. 11, 3 (2018), 16-30. To Appear.

[26] S. Liao, On the homotopy analysis method for nonlinear problems, Appl. Math. Comput. 147 (2004), 499-513.

[27] S. Nanda, On integration of fuzzy mappings, Fuzzy Sets and Systems, 32 (1989), 95-101.

[28] M. Porshokouhi, B. Ghanbari, Variational iteration method for solving Volterra and Fredholm integral equations of the second kind, Gen. Math. Notes, 2 (2011), 143-148.

[29] Y. Shao, H. Zhang, Fuzzy integral equations and strong fuzzy Henstock integrals, Abstr. Appl. Anal. (2014), 1-8.

[30] A. Wazwaz, A new algorithm for calculationg Adomian polynomials for nonlinear operators, Appl. Math. Comput. 3 (2000), 53-69.

Ahmed A. Hamoud

Department of Mathematics, Taiz University, Taiz, 96704, Yemen.

Research Scholar at. Department of Mathematics, Dr. Babasaheb Ambedkar Marathwada University, Aurangabad, 431004, India.

email: drahmedselwi985@gmail.com

Kirtiwant P. Ghadle

Department of Mathematics, Dr. Babasaheb Ambedkar Marathwada University, Aurangabad, 431004, India.

email:drkp.ghadle@gmail.com 